\title{
Comprehensive Analysis of Clinicopathologic Factors Predictive of an Unfavorable Prognosis in Patients With Acinic Cell Carcinoma of the Parotid Gland
}

\author{
Young Min Park ${ }^{1}$ (i) $\cdot$ Sun Och Yoon ${ }^{2} \cdot$ Joo Hyun Kim ${ }^{1} \cdot$ Min Seok Kang ${ }^{1} \cdot$ Da Hee Kim $\cdot$ Yoon Woo Koh ${ }^{1}$ \\ Se-Heon Kim${ }^{1} \cdot$ Jae-Yol Lim ${ }^{1}$ (D) $\cdot$ Eun Chang Choi ${ }^{1}$ (D) \\ Departments of ${ }^{1}$ Otorhinolaryngology and ${ }^{2}$ Pathology, Yonsei University College of Medicine, Seoul, Korea
}

Objectives. In subset of patients, acinic cell carcinoma (AcCC) exhibits aggressive features such as recurrence, distant metastasis, and mortality. This study aimed to investigate clinicopathologic factors influencing patients' prognosis and to identify adverse features predictive of an unfavorable prognosis.

Methods. Between January 2000 and December 2016, 59 patients with AcCC were enrolled in this study.

Results. The patients' 5-year overall survival rate was $93.3 \%$, and their 5-year recurrence-free survival rate was $80.5 \%$. During the study period, recurrence occurred in 10 patients. The mean time to recurrence after surgery was 26 months (range, 5-60 months). During the study period, three patients died from the disease. Univariate analysis showed that sex, surgical extent, extranodal extension, T classification, and TNM stage were significantly associated with disease recurrence. Multivariate analysis showed that, among the clinicopathologic factors included in the analysis, only TNM stage displayed a statistically significant correlation with disease recurrence.

Conclusion. Surgical treatment alone yielded good results for AcCC, and additional treatment did not affect the recurrencefree survival rate or the overall survival rate, even when the resection margin was less than $1 \mathrm{~mm}$. Other pathologic factors did not show prognostic significance for disease recurrence or death.

Keywords. Acinic Cell Carcinoma; Parotid Gland; Prognostic Factor

\section{INTRODUCTION}

Acinic cell carcinoma (AcCC) is a rare salivary gland malignancy that accounts for approximately $12 \%$ to $17 \%$ of all salivary gland

\footnotetext{
- Received September 16, 2019

Revised October 27, 2019

Accepted December 18, 2019

- Corresponding author: Jae-Yol Lim

Department of Otorhinolaryngology, Gangnam Severance Hospital, Yonsei

University College of Medicine, 211 Eonju-ro, Gangnam-gu, Seoul 06273,

Korea

Tel: +82-2-2019-3460, Fax: +82-2-3463-4750

E-mail: jylimmd@yuhs.ac

- Co-Corresponding author: Eun Chang Choi

Department of Otorhinolaryngology, Gangnam Severance Hospital, Yonsei

University College of Medicine, 211 Eonju-ro, Gangnam-gu, Seoul 06273,

Korea

Tel: +82-2-2228-3600, Fax: +82-2-393-0580

E-mail: eunchangmd@yuhs.ac
}

cancers. Females display a higher incidence of this disease, with a female-to-male incidence ratio of 1.36:1, and its overall incidence is increasing, especially among males [1]. This neoplasm mostly presents as a slow-growing parotid mass, and is generally considered to be a low-grade malignancy. Surgery alone is generally linked to excellent long-term disease control [2]. However, in a subset of patients, AcCC exhibits aggressive features such as recurrence, distant metastasis, and mortality. Previous research pointed to aggressive histologic variants of $\mathrm{AcCC}$, a minority of which could transform into high-grade malignancy, but the clinicopathologic predictive factors of a poor prognosis have not yet been fully elucidated, due to the rarity of the disease and the heterogeneity of treatments in study populations. Adjuvant radiotherapy is generally recommended for cases with adverse pathologic features, but the benefits of postoperative radiotherapy for disease control and/or survival in patients with low-grade salivary gland cancers remain controversial [3-6]. The purpose of

Copyright @ 2021 by Korean Society of Otorhinolaryngology-Head and Neck Surgery.

This is an open-access article distributed under the terms of the Creative Commons Attribution Non-Commercial License (https://creativecommons.org/licenses/by-nc/4.0)

which permits unrestricted non-commercial use, distribution, and reproduction in any medium, provided the original work is properly cited. 
this study was to investigate the treatment outcomes of patients with AcCC of the parotid gland, to analyze the clinicopathologic factors that influenced treatment outcomes, and to identify adverse features predictive of an unfavorable prognosis. We also assessed the influence of surgical extent and adjuvant therapy on treatment outcomes in patients with AcCC of the parotid gland.

\section{MATERIALS AND METHODS}

The Institutional Review Board of Yonsei University approved this study (IRB No. 2017-0037-001) and Informed consent was waived.

Among patients who were diagnosed with AcCC and visited our hospital from January 2000 to December 2016, we enrolled 59 patients who underwent surgery as an initial treatment and conducted a retrospective review of their medical records. Inclusion criteria were as follows: (1) patients diagnosed with AcCC of the parotid gland after surgery; (2) patients who underwent surgery with curative intent as initial treatment; and (3) patients who underwent at least 12 months of follow-up. Exclusion criteria were as follows: (1) distant metastasis at the time of diagnosis; and (2) previous surgery or radiotherapy on the head and neck. Our study included 29 males and 30 females. The patients' ages ranged from 6 to 72 year, and the mean age was 41.2 years. The relationship between clinicopathologic parameters (sex, age, tumor size, TNM stage, surgical extent, adjuvant radiotherapy, resection margin status, intraglandular lymph node metastasis, and histologic grade) and unfavorable outcomes (recurrence or death) were analyzed. Pathologic TNM stage was determined based on pathology reports using the 8th American Joint Committee on Cancer Staging Manual. Inclusion criteria were as follows: histologically diagnosed as only AcCC, and cases with sufficient clinicopathological information available from medical records. Exclusion criteria were as follows: distant metastasis at the time of presentation, and loss to follow-up.

The extent of parotidectomy was determined according to the location and extent of the disease, as determined by preoperative imaging studies. The extent of surgery was defined as superficial

\section{H I G H L I G H T S}

- The 5-year overall survival rate in 59 patients with acinic cell carcinoma (AcCC) was 93.3\%, and their 5-year recurrencefree survival rate was $80.5 \%$.

- Multivariate analysis showed that only TNM stage displayed a statistically significant correlation with disease recurrence.

- Surgical treatment alone yielded good results for AcCC, and additional treatment did not affect the recurrence-free survival rate or the overall survival rate. or total parotidectomy. Elective neck dissection was not usually performed in the absence of cervical lymph node metastasis; and, if necessary, selective neck dissection, including levels II and III, was performed. In the case of cervical lymph node metastasis, modified radical neck dissection, including levels II-V, was performed. In postoperative pathologic examination, adjuvant treatment was considered in cases of advanced $\mathrm{T}$ stage, positive margin, extracapsular nodal spread, multiple metastatic lymph nodes, lymphovascular invasion, and perineural invasion.

Patients' clinical information, tumor location and stage, pathologic findings, recurrence, date of recurrence, recurrence pattern, death events, date of death, and cause of death were collected and analyzed. Patients were categorized into either favorable or unfavorable groups according to their prognosis. We defined unfavorable group as local, regional, distant failure cases, or death related to the disease during follow-up period. For unfavorable group, available pathology materials, tumor growth pattern, tumor border, peritumoral stroma, cytological features, tumor necrosis, lymphovascular invasion, perineural invasion, and high grade transformation were additionally reviewed. Univariate and multivariate analyses were performed to evaluate the prognostic factors affecting patients' survival. Chi-square or Fisher's exact tests were used to evaluate the differences in categorical variables between two independent groups. An independent two-sample $t$-test was used to assess differences in continuous variables between the two independent groups. Multivariate logistic regression analysis was performed to model dichotomous variables. Kaplan-Meier curves were used to analyze disease-free survival, and survival outcomes were assessed using log-rank test. A $P$-value $<0.05$ indicated statistical significance. Statistical analyses were performed using SPSS ver. 18.0 (SPSS Inc., Chicago, IL, USA).

\section{RESULTS}

Fifty-nine patients were enrolled in this study. AcCC was found in the left parotid gland in 24 patients and in the right parotid gland in 35 patients. All patients underwent surgery with curative intent as the initial treatment; 31 underwent superficial parotidectomy and 28 underwent total parotidectomy. Elective selective neck dissection, including levels II and III, was performed in eight patients, and two patients received therapeutic modified radical neck dissection. Twenty-eight patients had TNM stage I disease, 16 patients had stage II disease, six patients had stage III disease, and nine patients had stage IV disease (Table 1).

The mean follow-up period was 52 months (range, 8-203 months). The 5-year overall survival rate was $93.3 \%$, and the 5 -year recurrence-free survival rate was $80.5 \%$ (Fig. 1). During the study period, recurrence took place in 10 patients (eight cases of local recurrence, three cases of regional recurrence, and two cases of distant metastasis). The mean time to recurrence after surgery 
Table 1. Clinical information of all patients with acinic cell carcinoma of the parotid gland

\begin{tabular}{|c|c|}
\hline Variable & Value $(n=59)$ \\
\hline Age (yr) & $41.2(6-72)$ \\
\hline \multicolumn{2}{|l|}{ Sex } \\
\hline Male & $29(49.2)$ \\
\hline Female & $30(50.8)$ \\
\hline \multicolumn{2}{|l|}{ Lesion side } \\
\hline Left & $24(40.7)$ \\
\hline Right & 35 (59.3) \\
\hline Bilateral & 0 \\
\hline \multicolumn{2}{|c|}{ Extent of surgery for parotid } \\
\hline Superficial & $31(52.5)$ \\
\hline Total & $28(47.5)$ \\
\hline \multicolumn{2}{|l|}{ Neck dissection } \\
\hline None & $49(83.0)$ \\
\hline SND including II-III & $8(13.6)$ \\
\hline MRND & $2(3.4)$ \\
\hline \multicolumn{2}{|l|}{ Adjuvant therapy } \\
\hline No & $43(72.9)$ \\
\hline Yes & $16(27.1)$ \\
\hline \multicolumn{2}{|l|}{ pT classification } \\
\hline 1 & 23 \\
\hline 2 & 27 \\
\hline 3 & 2 \\
\hline 4 & 7 \\
\hline \multicolumn{2}{|l|}{ pN classification } \\
\hline 0 & 54 \\
\hline 1 & 3 \\
\hline 2 & 2 \\
\hline \multicolumn{2}{|l|}{ TNM stage } \\
\hline I & $28(47.5)$ \\
\hline ॥ & $16(27.1)$ \\
\hline III & $6(10.2)$ \\
\hline IV & $9(15.3)$ \\
\hline \multicolumn{2}{|l|}{ Recurrence } \\
\hline Local 8 & $11(18.6)$ \\
\hline Regional 3 & $5(8.5)$ \\
\hline Distant metastasis 2 & $2(3.4)$ \\
\hline
\end{tabular}

Values are presented as mean (range) or number (\%).

SND, selective neck dissection; MRND, modified radical neck dissection. was 26 months (range, 5-60 months). Nine patients experienced recurrence within 36 months after surgery, except for one patient who experienced recurrence 60 months postoperatively. Nine patients underwent salvage surgery and one patient received concurrent chemoradiotherapy to control recurrent disease. During
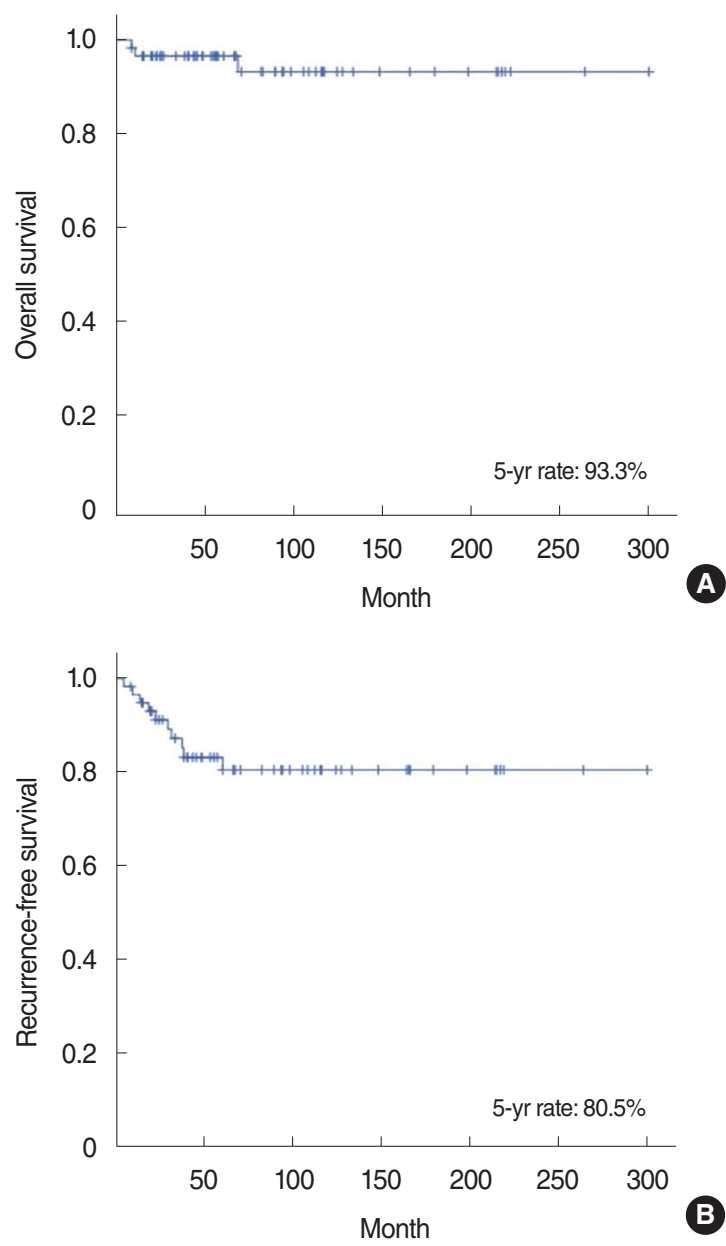

Fig. 1. Five-year overall survival $(A)$ and 5-year recurrence-free survival (B) among all patients.
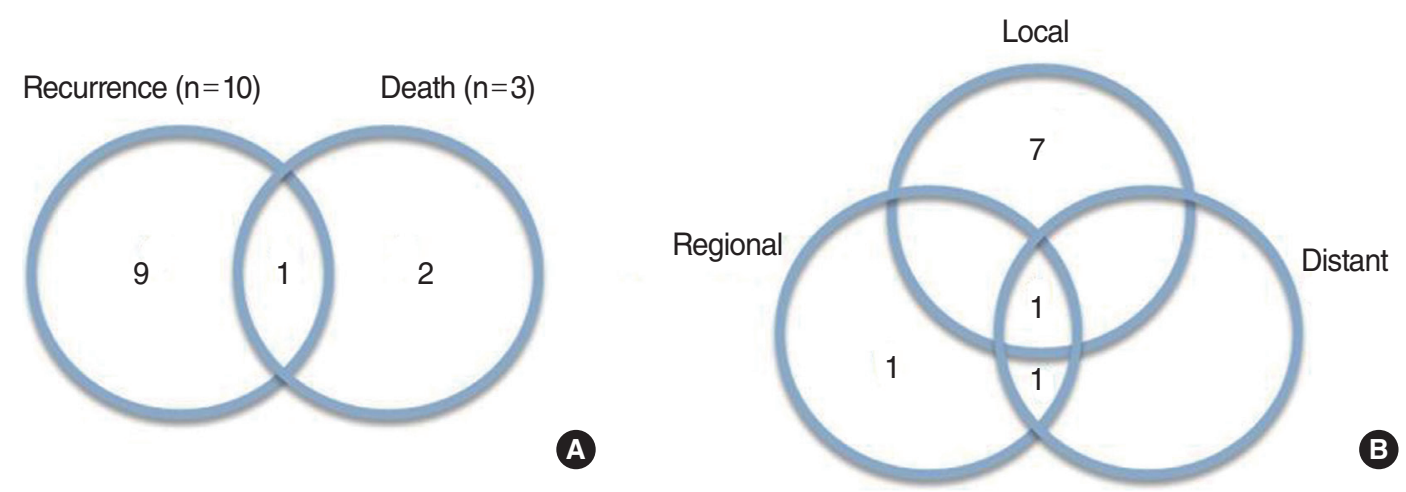

Fig. 2. Disease recurrence and death events in patients with acinic cell carcinoma of the parotid gland after surgery. (A) Disease recurrence and death events. (B) Disease recurrence pattern. 
the study period, only three patients died due to the disease (Fig. 2).

A univariate analysis was conducted of the clinicopathologic factors associated with recurrence (Table 2$)$. Sex $(P=0.041)$, surgical extent $(P=0.020)$, extranodal extension $(P<0.001)$, T classification $(P=0.023)$, and TNM stage $(P<0.001)$ showed statistically significant associations with disease recurrence. Age, resection margin status, lymph node metastasis, lymphovascular invasion, perineural invasion, and adjuvant treatment were not significantly correlated with disease recurrence. Kaplan-Meier analysis was used to evaluate the 5-year recurrence-free survival rate. Statistically significant differences in the survival rate were found

Table 2. Univariate analysis of clinical variables for RFS

\begin{tabular}{|c|c|c|c|c|}
\hline Variable & $\begin{array}{c}\text { No. of } \\
\text { patients (\%) }\end{array}$ & $\begin{array}{l}\text { No. of } \\
\text { events }\end{array}$ & $\begin{array}{c}\text { 5-Year } \\
\text { RFS (\%) }\end{array}$ & $P$-value \\
\hline Age (yr) & & & & 0.818 \\
\hline$<60$ & $48(81.4)$ & 8 & 81.2 & \\
\hline$\geq 60$ & $11(18.6)$ & 2 & 77.1 & \\
\hline Sex & & & & 0.041 \\
\hline Male & $29(49.2)$ & 8 & 68.9 & \\
\hline Female & $30(50.8)$ & 2 & 92.6 & \\
\hline Surgical extent & & & & 0.020 \\
\hline Superficial & $31(52.5)$ & 2 & 93.1 & \\
\hline Total & $28(47.5)$ & 8 & 64.8 & \\
\hline Resection margin & & & & 0.583 \\
\hline Positive & $4(6.8)$ & 1 & 75.0 & \\
\hline Negative & $55(93.2)$ & 9 & 81.1 & \\
\hline Margin status & & & & 0.112 \\
\hline Positive & $4(6.8)$ & 1 & 75.0 & \\
\hline Close margin (<1 mm) & $18(30.5)$ & 5 & 63.6 & \\
\hline Negative & $37(62.7)$ & 4 & 88.1 & \\
\hline LN metastasis & & & & 0.329 \\
\hline Yes & $4(6.8)$ & 1 & 75.0 & \\
\hline No & $55(93.2)$ & 9 & 81.3 & \\
\hline ENE & & & & $<0.001$ \\
\hline Yes & $1(1.7)$ & 1 & 0 & \\
\hline No & $58(98.3)$ & 9 & 82.0 & \\
\hline LVI & & & & 0.641 \\
\hline Yes & $4(6.8)$ & 1 & 66.7 & \\
\hline No & 55 (93.2) & 9 & 81.3 & \\
\hline $\mathrm{PNI}$ & & & & 0.249 \\
\hline Yes & $2(3.4)$ & 1 & 50.0 & \\
\hline No & 57 (96.6) & 9 & 81.9 & \\
\hline Adjuvant Tx & & & & 0.659 \\
\hline Yes & $16(27.1)$ & 2 & 85.7 & \\
\hline No & $43(72.9)$ & 8 & 78.6 & \\
\hline T classification & & & & 0.023 \\
\hline $1 \& 2$ & $50(84.7)$ & 5 & 86.4 & \\
\hline $3 \& 4$ & $9(15.3)$ & 4 & 50.0 & \\
\hline TNM stage & & & & $<0.001$ \\
\hline | \& || & $44(74.6)$ & 2 & 92.8 & \\
\hline III \& IV & $15(25.4)$ & 7 & 40.6 & \\
\hline
\end{tabular}

RFS, recurrence-free survival; LN, lymph node; ENE, extranodal extension; LVI, lymphovascular invasion; PNI, perineural invasion; Tx, treatment. according to sex, TNM stage, and T classification (Fig. 3). The clinicopathologic factors related to recurrence were then entered into a multivariate analysis. Of the clinicopathologic factors included in the analysis, only TNM stage showed a statistically significant correlation with disease recurrence $(P=0.018)$ (Table 3$)$.
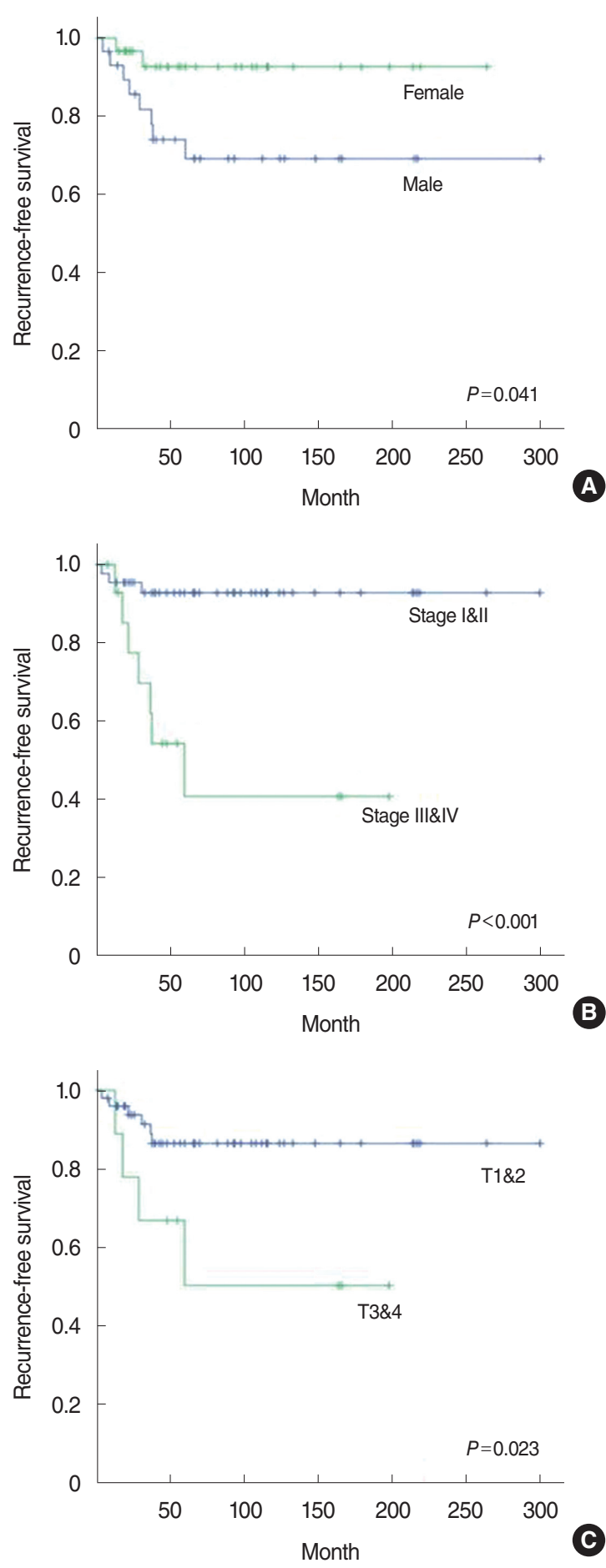

Fig. 3. Kaplan-Meier analysis of 5-year recurrence-free survival according to sex (A), TNM stage (B), and T classification (C). 
Table 3. Multivariate Cox regression analysis for RFS and OS adjusting for various prognostic factors

\begin{tabular}{lrll}
\hline Factor & Hazard ratio & \multicolumn{1}{c}{$95 \% \mathrm{Cl}$} & $P$-value \\
\hline Associated with RFS & & & \\
Sex & 0.402 & $0.077-2.094$ & 0.279 \\
Resection margin & 2.358 & $0.419-13.274$ & 0.331 \\
Extranodal extension & 115.635 & $0.000-1.123$ & 0.958 \\
PNI & 0.500 & $0.047-5.315$ & 0.565 \\
T classification & 0.654 & $0.365-1.170$ & 0.152 \\
TNM stage & 2.312 & $1.156-4.623$ & 0.018 \\
Associated with OS & & & \\
Age & 1.204 & $0.579-2.503$ & 0.619 \\
Sex & 0.004 & $0.000-1055$ & 0.679 \\
LVI & 12.571 & $0.000-4.998$ & 0.982 \\
PNI & 65.845 & $0.000-1.592$ & 0.971 \\
T classification & 7.385 & $0.000-4.684$ & 0.894 \\
TNM stage & 1.723 & $0.000-1735$ & 0.954 \\
\hline
\end{tabular}

RFS, recurrence-free survival; OS, overall survival; $\mathrm{Cl}$, confidence interval; $\mathrm{PNI}$, perineural invasion; LVI, lymphovascular invasion.

Clinicopathologic factors associated with death events were analyzed by univariate analysis (Table 4$)$. There was a statistically significant correlation between age $(P=0.020)$, perineural invasion ( $P=0.005)$, T classification $(P=0.006)$,TNM stage $(P=0.001)$, and death. Sex, surgical extent, resection margin, lymph node metastasis, extranodal extension, lymphovascular invasion, and adjuvant treatment were not correlated with death. Kaplan-Meier analysis was used to examine the 5-year overall survival rate of patients, and statistically significant differences were found according to TNM stage and T classification (Fig. 4). Clinicopathologic factors related to death events were analyzed by multivariate analysis, and no statistically significant correlations were found (Table 3).

\section{Surgical margin status}

A positive margin was defined as the presence of cancer cells in the margin of the resected specimen, and a negative margin was defined as the absence of cancer cells. Positive margins were reported in four patients, and negative margins were reported in the remaining 55 patients. A statistical analysis of the relationship of margin status with recurrence and death events showed no significant correlation. Due to the presence of the facial nerve within the parotid gland, surgeons often receive a pathologic report with a margin of less than $1 \mathrm{~mm}$, known as an "abutting" margin status [7]. When these "abutting" margins were regarded as positive, a positive margin was found to be significantly correlated with disease recurrence in the univariate analysis $(P=0.038)$, but not in the multivariate analysis.

\section{Surgical extent and adjuvant treatment}

We classified the extent of surgery as superficial parotidectomy or total parotidectomy, and evaluated possible relationships associated with disease recurrence or death. Surgical extent was
Table 4. Univariate analysis of clinical variables for OS

\begin{tabular}{|c|c|c|c|c|}
\hline Variable & $\begin{array}{c}\text { No. of } \\
\text { patients (\%) }\end{array}$ & $\begin{array}{l}\text { No. of } \\
\text { events }\end{array}$ & $\begin{array}{l}5-Y e a r \\
\text { OS (\%) }\end{array}$ & $P$-value \\
\hline Age (yr) & & & & 0.020 \\
\hline$<60$ & $48(81.4)$ & 8 & 97.9 & \\
\hline$\geq 60$ & $11(18.6)$ & 2 & 68.2 & \\
\hline Sex & & & & 0.080 \\
\hline Male & $29(49.2)$ & 8 & 86.8 & \\
\hline Female & $30(50.8)$ & 2 & 100 & \\
\hline Surgical extent & & & & 0.446 \\
\hline Superficial & $31(52.5)$ & 2 & 96.8 & \\
\hline Total & $28(47.5)$ & 8 & 88.4 & \\
\hline Resection margin & & & & 0.608 \\
\hline Positive & $4(6.8)$ & 1 & 100 & \\
\hline Negative & 55 (93.2) & 9 & 92.6 & \\
\hline Margin status & & & & 0.829 \\
\hline Positive & $4(6.8)$ & 1 & 100 & \\
\hline Close margin $(<1 \mathrm{~mm})$ & $18(30.5)$ & 5 & 66.7 & \\
\hline Negative & $37(62.7)$ & 4 & 94.6 & \\
\hline LN metastasis & & & & 0.720 \\
\hline Yes & $4(6.8)$ & 1 & 100 & \\
\hline No & 55 (93.2) & 9 & 93.0 & \\
\hline ENE & & & & 0.790 \\
\hline Yes & $1(1.7)$ & 1 & 100 & \\
\hline No & $58(98.3)$ & 9 & 93.1 & \\
\hline LVI & & & & 0.107 \\
\hline Yes & $4(6.8)$ & 1 & 66.7 & \\
\hline No & 55 (93.2) & 9 & 96.3 & \\
\hline $\mathrm{PNI}$ & & & & 0.005 \\
\hline Yes & $2(3.4)$ & 1 & 0 & \\
\hline No & 57 (96.6) & 9 & 96.5 & \\
\hline Adjuvant Tx & & & & 0.306 \\
\hline Yes & $16(27.1)$ & 2 & 100 & \\
\hline No & 43 (72.9) & 8 & 91.0 & \\
\hline T classification & & & & 0.006 \\
\hline $1 \& 2$ & $50(84.7)$ & 5 & 96.2 & \\
\hline $3 \& 4$ & $9(15.3)$ & 4 & 77.8 & \\
\hline TNM stage & & & & 0.001 \\
\hline |\& || & $44(74.6)$ & 2 & 100 & \\
\hline III \& IV & $15(25.4)$ & 7 & 71.8 & \\
\hline
\end{tabular}

OS, overall survival; LN, lymph node; ENE, extranodal extension; LVI, lymphovascular invasion; PNI, perineural invasion; Tx, treatment.

significantly correlated $(P=0.020)$ with disease recurrence in the univariate analysis, but not in the multivariate analysis. There were no statistically significant correlations between adjuvant treatment and disease recurrence or patient mortality.

\section{Unexpected malignancy}

All patients underwent fine-needle aspiration cytology (FNAC) before surgery. Forty-five patients were suspected to have AcCC or malignancy according to the preoperative cytology report, but the remaining 14 patients underwent surgery without suspicion of malignancy before surgery. All tumors in these patients were stage $\mathrm{T} 1$ orT2, and a negative margin was obtained in 13 of these 

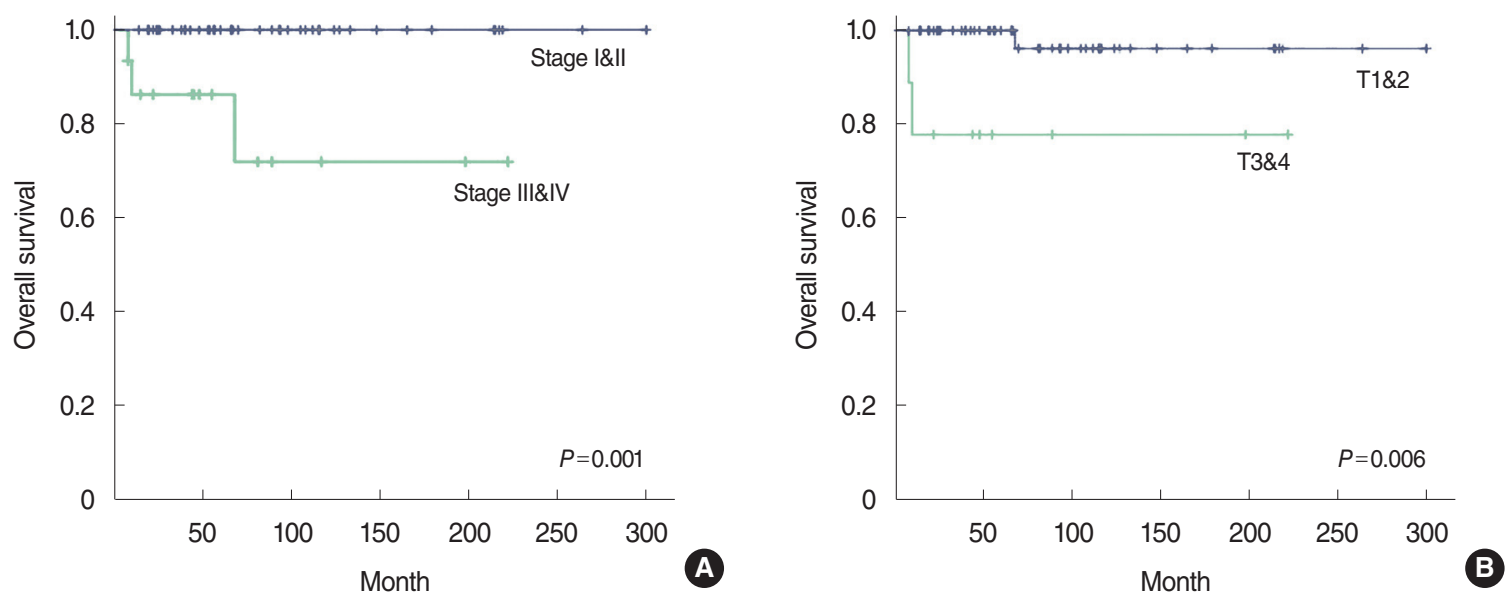

Fig. 4. Kaplan-Meier analysis of 5-year overall survival according to TNM stage (A) and T classification (B).

Table 5. Pathologic features for patients group with disease recurrence

\begin{tabular}{|c|c|c|c|c|c|c|}
\hline Case & Growth pattern & Major pattern & Capsule invasion & Stroma & High grade transformation & Necrosis \\
\hline 1 & Multinodular & Solid & Yes & Sclerosis & No & No \\
\hline 2 & Multinodular & Solid & No & Sclerosis & No & No \\
\hline 3 & Single round & Solid & Yes & Sclerosis & No & No \\
\hline 4 & Multinodular & Papillocystic & Yes & Sclerosis & No & No \\
\hline 5 & Single round & Papillocystic & Yes & None & No & No \\
\hline 6 & Infiltrative & Microcystic & Yes & Sclerosis & No & No \\
\hline 7 & Single round & Papillocystic & No & None & No & No \\
\hline 8 & Multinodular & Solid & No & Sclerosis & No & No \\
\hline 9 & Single round & Papillocystic & No & Sclerosis & No & Yes \\
\hline 10 & Single round & Papillocystic & Yes & Sclerosis & No & No \\
\hline
\end{tabular}

14 patients after surgery. Only two patients experienced recurrence during the study, but they survived without residual disease after salvage surgery (one local recurrence and one regional recurrence).

\section{Salvage treatment}

Among the 10 patients who had disease recurrence, salvage surgery was performed in eight patients, followed by disease-free survival. The remaining two patients had both distant metastasis and loco-regional recurrence; both received concurrent chemotherapy, and one died during the study period.

\section{Pathologic features specific to unfavorable outcomes}

The pathologic features associated with unfavorable outcomes, based on the available pathology materials $(n=10)$, are summarized in Table 5. Within this group, pathologic features varied in terms of tumor growth pattern, tumor border, peritumoral stroma, cytological features, tumor necrosis, and high-grade transformation. Interestingly, high-grade transformation was not observed in any cases in our study, and other histopathologic features were likewise not found to be characteristic of unfavorable outcomes.

\section{DISCUSSION}

AcCC is a rare disease entity that occurs in the major salivary glands, accounting for about $10 \%$ of salivary gland tumors [8]. It occurs in relatively young patients, and shows less aggressive features than other salivary carcinomas. AcCC is generally known as a low-grade salivary gland tumor, but its prognosis is sometimes poor in a subset of AcCC patients in whom pathologically high-grade features are observed [9]. Neskey et al. [10] reported that several adverse prognostic factors, including sex, age, a tumor size larger than $3 \mathrm{~cm}$, and distant metastasis, were associated with poorer survival. Although adjuvant radiotherapy is generally recommended in patients with several adverse features, the indications for adjuvant treatment in patients with AcCC have not yet been established [11]. In particular, whether adjuvant treatment is indicated in young patients with a long life expectancy after treatment is an important issue, as quality of life can deteriorate after intensive treatment with radiotherapy or concurrent chemoradiotherapy.

Furthermore, more than $90 \%$ of AcCCs are known to occur in the parotid glands, while fewer than $10 \%$ occur in the minor salivary gland, and the condition rarely occurs in the subman- 
dibular gland. Different concerns are associated with the surgical treatment of carcinoma arising from the parotid gland, compared to cancer in the minor salivary or submandibular gland. Since the facial nerve is present inside the parotid gland, and the anatomy around the parotid gland is complex, it is challenging to obtain a sufficient safety margin when the tumor is resected. As the surgeon should both preserve the facial nerve and extirpate the tumor with a sufficient margin during the operation, a dilemma is encountered in real clinical settings. Accordingly, salivary gland carcinoma-although histologically a single type of carcinoma-has unique clinical characteristics according to the gland in which it originates. Therefore, this study focused only on AcCCs in the parotid gland.

In our study, the mean age of patients with $\mathrm{AcCC}$ was 41 years (6-72 years). The 5-year overall survival rate was $93 \%$, and the 5 -year disease-free survival rate was $80 \%$. No statistically significant correlations were found between death events and any clinicopathologic factors, and TNM stage was the only parameter significantly associated with disease recurrence. During the study period, 10 patients experienced disease recurrence, of whom eight patients survived in a disease-free state after successful salvage surgery. Even in cases of recurrence, most of the patients $(80 \%)$ were able to undergo salvage surgery, and the oncologic results were also favorable after salvage treatment.

In this study, 18 patients showed a resection margin of less than $1 \mathrm{~mm}$, also known as an "abutting" margin status. Eight of these patients received adjuvant radiotherapy, and the remaining 10 patients did not receive adjuvant therapy; however, there was no significant difference in disease recurrence or mortality between patients who received adjuvant treatment and those who did not. Generally, from the perspective of an oncologic surgeon, a resection margin of less than $1 \mathrm{~mm}$ is regarded as a positive margin and adjuvant therapy should be considered. However, for AcCC, it was confirmed that local control was good without adjuvant therapy in cases with an "abutting" margin.

In salivary gland cancer, histologic grade and tumor type are important prognostic parameters, together with other risk factors such as TNM stage, resection margin status, and lymph node metastasis. Biron et al. [3] performed a retrospective survival analysis of 2,061 patients based on the Surveillance, Epidemiology, and End Results registry, and suggested that histologic grade is a stronger predictor of survival than TNM classification in AcCC patients. However, a standard grading scheme for salivary gland cancer remains to be established, and adverse pathologic factors predictive of prognosis also remain unclear. Therefore, in this study, we intensively investigated the pathologic factors that could predict the prognosis of AcCC patients; however, no pathologic feature showed a significant relationship with disease recurrence or death. Interestingly, high-grade transformation findings known to be associated with a poor prognosis were not observed in our study.

FNAC is usually performed before surgery in patients with salivary gland tumors to determine whether the tumor is malig- nant. However, a low-grade salivary gland malignancy may be unexpectedly diagnosed as malignant on a permanent pathologic examination after surgery, inconsistent with the results of preoperative FNAC. In these unexpected cases, the surgical margin status might be inappropriate. Especially in parotid surgery, since the facial nerve runs within the parotid gland, the tumor could be marginally excised to preserve the facial nerve. In cases in which the tumor is excised marginally without a sufficient safety margin, the surgeon must consider whether to perform a completion parotidectomy or to perform adjuvant treatment. To overcome the limitation of the false-negative rate of FNAC, Cha et al. [12] reported that intraoperative frozen section examinations may be helpful in the diagnosis of AcCC of the parotid gland. However, intraoperative frozen sections are not usually performed during parotid surgery in real clinical situations. In our study, 14 patients $(23.7 \%)$ were unexpectedly diagnosed with AcCC after surgery, inconsistent with their preoperative FNAC results. Of these, 12 patients underwent only superficial parotidectomy, while the remaining two patients received total parotidectomy. Of these 12 patients, only one patient received adjuvant radiotherapy. Nonetheless, disease only recurred in two patients during the study period, irrespective of the extent of surgery and adjuvant therapy, and the two patients with recurrent disease underwent successful salvage surgery and survived in a disease-free state.

Based on our results, surgical treatment alone yielded extremely good results for AcCC, and surgical extent and adjuvant therapy did not affect the disease-free survival or overall survival rates, even when the resection margin was less than $1 \mathrm{~mm}$. The risk of disease recurrence was only higher in $\mathrm{AcCC}$ with an advanced TNM stage, in which more aggressive treatment, such as total parotidectomy or adjuvant radiotherapy, may be considered to prevent disease recurrence.

\section{CONFLICT OF INTEREST}

No potential conflict of interest relevant to this article was reported.

\section{ORCID}

Young Min Park https://orcid.org/0000-0002-7593-8461

Jae-Yol Lim https://orcid.org/0000-0002-9757-6414

Eun Chang Choi https://orcid.org/0000-0002-9631-8157

\section{AUTHOR CONTRIBUTIONS}

Conceptualization:YMP. Data curation: SOY, MSK. Formal analysis: JHK. Methodology: DHK. Project administration:YWK.Vi- 
sualization: SHK. Writing - original draft: JYL. Writing - review \& editing: ECC.

\section{REFERENCES}

1. Patel NR, Sanghvi S, Khan MN, Husain Q, Baredes S, Eloy JA. Demographic trends and disease-specific survival in salivary acinic cell carcinoma: an analysis of 1129 cases. Laryngoscope. 2014 Jan;124(1): $172-8$.

2. Gomez DR, Katabi N, Zhung J,Wolden SL, Zelefsky MJ, Kraus DH, et al. Clinical and pathologic prognostic features in acinic cell carcinoma of the parotid gland. Cancer. 2009 May;115(10):2128-37.

3. Biron VL, Lentsch EJ, Gerry DR, Bewley AF. Factors influencing survival in acinic cell carcinoma: a retrospective survival analysis of 2061 patients. Head Neck. 2015 Jun;37(6):870-7.

4. Cho JK, Lim BW, Kim EH, Ko YH, Oh D, Noh JM, et al. Low-grade salivary gland cancers: treatment outcomes, extent of surgery and indications for postoperative adjuvant radiation therapy. Ann Surg Oncol. 2016 Dec;23(13):4368-75.

5. Richter SM, Friedmann P, MouradWF, Hu KS, Persky MS, Harrison LB. Postoperative radiation therapy for small, low-/intermediate-grade parotid tumors with close and/or positive surgical margins. Head Neck. 2012 Jul;34(7):953-5.
6. Bakst RL, SuW, Ozbek U, Knoll MA, Miles BA, GuptaV, et al.Adjuvant radiation for salivary gland malignancies is associated with improved survival: a National Cancer Database analysis. Adv Radiat Oncol. 2017 Apr;2(2):159-66.

7. Stodulski D, Mikaszewski B, Majewska H,Wisniewski P, Stankiewicz C. Close surgical margin after conservative parotidectomy in early stage low-/intermediate-grade parotid carcinoma: outcome of watch and wait policy. Oral Oncol. 2017 May;68:1-4.

8. Hoffman HT, Karnell LH, Robinson RA, Pinkston JA, Menck HR. National Cancer Data Base report on cancer of the head and neck: acinic cell carcinoma. Head Neck. 1999 Jul;21(4):297-309.

9. Vander Poorten V, Triantafyllou A, Thompson LD, Bishop J, Hauben E, Hunt J, et al. Salivary acinic cell carcinoma: reappraisal and update. Eur Arch Otorhinolaryngol. 2016 Nov;273(11):3511-31.

10. Neskey DM, Klein JD, Hicks S, Garden AS, Bell DM, El-Naggar AK, et al. Prognostic factors associated with decreased survival in patients with acinic cell carcinoma. JAMA Otolaryngol Head Neck Surg. 2013 Nov;139(11):1195-202.

11. Andreoli MT, Andreoli SM, Shrime MG, Devaiah AK. Radiotherapy in parotid acinic cell carcinoma: does it have an impact on survival? Arch Otolaryngol Head Neck Surg. 2012 May;138(5):463-6.

12. ChaW, Kim MS, Ahn JC, Cho SW, SunwooW, Song CM, et al. Clinical analysis of acinic cell carcinoma in parotid gland. Clin Exp Otorhinolaryngol. 2011 Dec;4(4):188-92. 